\section{Nanoprobe Crosses Blood-Brain Barrier to Image and Treat Tumor}

Brain cancer is one of the hardest types of cancer to treat. Until now, no nanoparticle used for imaging has been able to cross the blood-brain barrier-an almost impenetrable barrier that protects the brain from infection-and specifically bind to braintumor cells. With current techniques, doctors inject dyes into the body and use drugs to temporarily open the blood-brain barrier, risking infection. Now, through use of a genetically engineered mouse model, M. Zhang and O. Veiseh of the University of Washington, J. Olson of Fred Hutchinson Cancer Research Center, R.G. Ellenbogen of the University of Washington and Seattle Children's Hospital and Regional Medical Center, and their colleagues have been able to illuminate brain tumors by injecting fluorescent nanoparticles into the bloodstream that safely cross the blood-brain barrier. The nanoparticles remained in mouse tumors for up to five days and did not show any evidence of damaging the blood-brain barrier, according to results published in the August 1 issue of Cancer
Research (DOI: 10.1158/0008-5472.CAN09-1157; p. 6200).

The nanoprobe is fabricated from an iron oxide nanoparticle coated with biocompatible polyethylene glycol-grafted chitosan copolymer. Crossing the bloodbrain barrier depends on the size of the particle, its lipid content, and the electric charge on the particle. The nanoparticle built by Zhang and her colleagues remains small in wet conditions. The particle was about $33 \mathrm{~nm}$ in diameter when wet, about a third the size of similar particles used in other parts of the body.

To specifically target tumor cells, the research team used chlorotoxin, a small peptide isolated from scorpion venom that many research groups are exploring for its tumor-targeting abilities. On the nanoparticle's surface, the researchers placed a near-infrared fluorophore for optical imaging, and binding sites that could be used for attaching other molecules.

Results showed the nanoparticles improved the contrast in both magnetic resonance imaging and optical imaging, which is used during surgery.

"Brain cancers are very invasive, differ- ent from the other cancers. They will invade the surrounding tissue and there is no clear boundary between the tumor tissue and the normal brain tissue," said Zhang, a professor of materials science and engineering.

Being unable to distinguish a boundary complicates the surgery. Severe cognitive problems are a common side effect.

"If we can inject these nanoparticles with infrared dye, they will increase the contrast between the tumor tissue and the normal tissue," Zhang said. "So during the surgery, the surgeons can see the boundary more precisely.

"We call it 'brain tumor illumination or brain tumor painting,," she said. "The tumor will light up."

Nano-imaging could also help with early cancer detection, Zhang said. Current clinical imaging techniques have a maximum resolution of $1 \mathrm{~mm}$. Nanoparticles could improve the resolution by a factor of 10 or more, allowing detection of smaller tumors and earlier treatment.

Future research will evaluate this nanoparticle's potential for treating tumors, Zhang said. She and colleagues
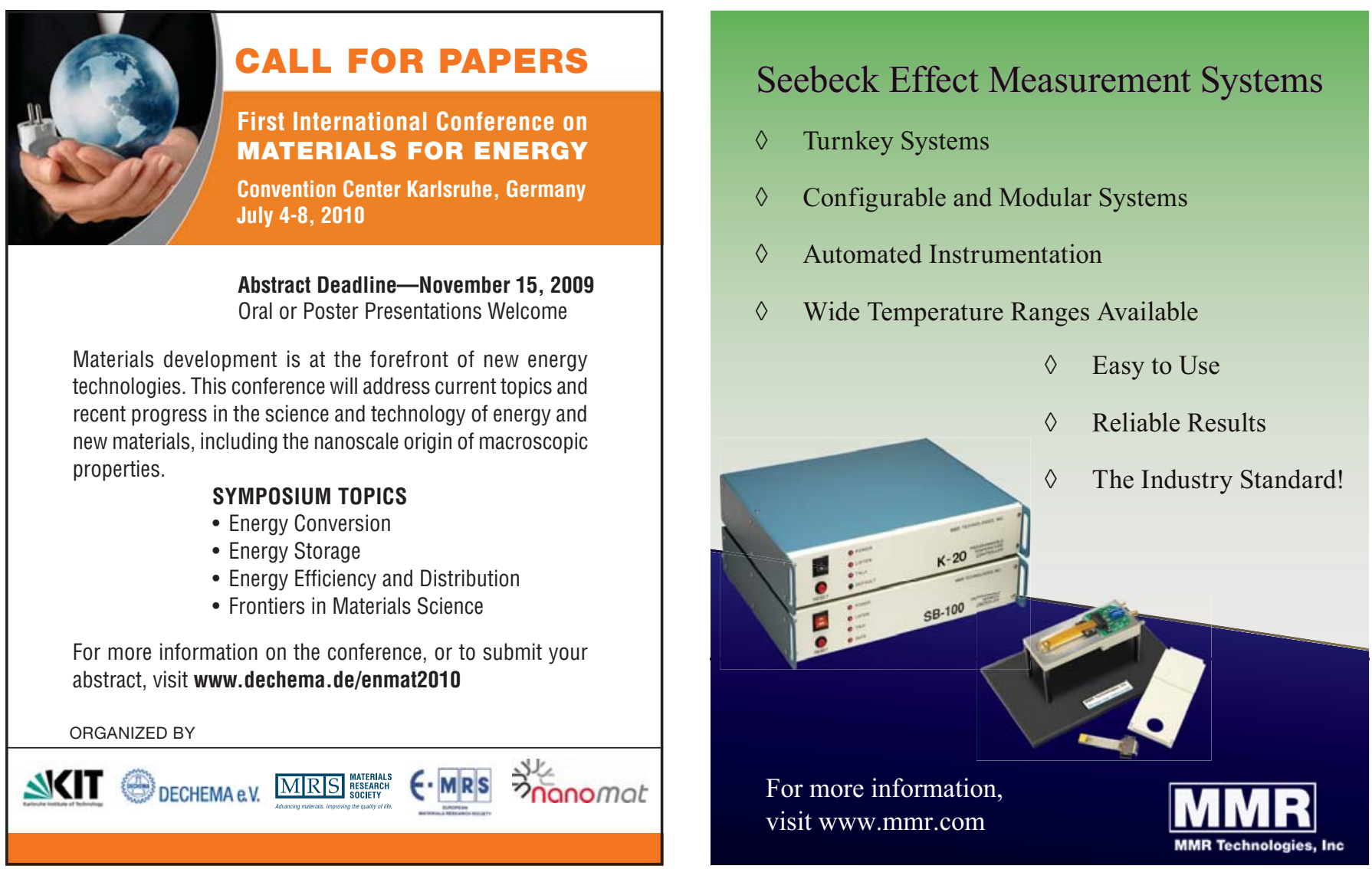
already showed that chlorotoxin combined with nanoparticles dramatically slows tumors' spread. They will see whether that ability could extend to brain cancer, the most common solid tumor to affect children.

Merely improving imaging, however, would improve patient outcomes.

"Precise imaging of brain tumors is phenomenally important. We know that patient survival for brain tumors is directly related to the amount of tumor that you can resect," said Ellenbogen, professor and chair of neurological surgery at the UW School of Medicine. "This is the next generation of cancer imaging," he said. "The last generation was CT, this generation was MRI, and this is the next generation of advances."

\section{LEGO Toy Serves as Model for Microfluidic Arrays}

A research team led by J. Frechette and G. Drazer of Johns Hopkins Institute for NanoBio Technology has used LEGO pieces to re-create microscopic activity taking place inside lab-on-a-chip devices at a scale they can more easily observe. As reported in the August 14 issue of Physical Review Letters (DOI:10.1103/ PhysRevLett.103.078301; \#078301), the research team constructed a square array using cylindrical LEGO pegs stacked two high and arranged in rows and columns on a LEGO board to create a lattice of obstacles. The board was attached to a Plexiglas sheet to improve its stiffness and pressed up against one wall of a Plexiglas tank filled with glycerol. Stainless steel balls of three different sizes, as well as plastic balls, were manually released from the top of the array; their paths to the bottom were tracked and timed with a camera.

"Microfluidic arrays are like miniature chemical plants," said Frechette, an assistant professor of chemical and biomolecular engineering in the university's Whiting School of Engineering. "One of the key components of these devices is the ability to separate one type of constituent from another. We investigated a microfluidic separation method that we suspected would remain the same when you scale it up from micrometers or nanometers to something as large as the size of billiard balls."

Graduate students M. Balvin and T. Iracki, and undergraduate student E. Sohn, all from the Department of Chemical and Biomolecular Engineering, performed multiple trials using each type of bead. They progressively rotated the board, increasing the relative angle between gravity and the columns of the array (i.e., altering the forcing angle). In doing so, they saw that the large balls did not move through the array in a diffuse or random manner as their small counterparts usually did in a microfluidic array. Instead, their paths were deterministic, meaning that they could be predicted with precision, said Drazer, also an assistant professor in the Whiting School of Engineering.

The researchers also noticed that the path followed by the balls was periodic once the balls were in motion and coincided with the direction of the lattice. As the forcing angle increased, some of the balls tended to shift over one, two, three, or as many as four pegs before continuing their vertical fall.

"Our experiment shows that if you know one single parameter - a measure of the asymmetry in the motion of a particle around a single obstacle-you can predict the path that particles will follow in a microfluidic array at any forcing angle, simply by doing geometry," Drazer said.
The fact that the balls moved in the same direction inside the array for different forcing angles is referred to as phase locking. If the array were to be scaled down to microor nanosize, the researchers said they would expect these phenomena to still be present and even increase depending on the factors such as the unavoidable irregularities of particle size or surface roughness.

"There are forces present between a particle and an obstacle when they get really close to each other which are present whether the system is at the micro- or nanoscale or as large as the LEGO board," Frechette said. "In this separation method, the periodic arrangement of the obstacles allows the small effect of these forces to accumulate, and amplify, which we suspect is the mechanism for particle separation."

This principle could be applied to the design of micro- or nanofluidic arrays, she said, so that they could be fabricated to "sort particles that had a different roughness, different charge, or different size. They should follow a different path in an array and could be collected separately."

Phase locking is likely to become less important, Drazer said, as the number of particles in solution becomes more concentrated.

"Next," he said, "we have to look at how concentrated your suspension can be before this principle is destroyed by particle-particle interactions."

\section{Upconversion Luminescence Found in $\mathrm{Yb}^{3+}, \mathrm{Tm}^{3+}$-Codoped $\gamma$-AION Phosphors}

Aluminum oxynitride (AlON) has been found to be a suitable phosphor matrix. Stokes luminescence properties of metalion-doped AlON have been studied recently. However, up to now, upconversion luminescence in an $\mathrm{AlON}$ phosphor has not been reported. Now, F. Zhang, L. An, X. Liu, G. Zhou, X. Yuan, and $S$. Wang at the Shanghai Institute of Ceramics of the Chinese Academy of Sciences have found that $\mathrm{Yb}^{3+}, \mathrm{Tm}^{3+}$-codoped $\mathrm{AlON}$ shows strong upconversion luminescence at optimal concentrations of $\mathrm{Yb}^{3+}$ and $\mathrm{Tm}^{3+}$.
The upconversion emission mechanism was also investigated.

As reported in the August issue of the Journal of the American Ceramics Society (DOI: 10.1111/j.1551-2916.2009.03131.x; p. 1888), Zhang and co-workers prepared $\gamma$-AlON matrix first by carbothermal reduction and nitridation processing using high purity $\mathrm{Al}_{2} \mathrm{O}_{3}$ and carbon black as precursors. Next, a series of $\mathrm{Yb}^{3+}$, $\mathrm{Tm}^{3+}$-codoped $\gamma$-AlON powders with $0.3-1.2 \mathrm{~mol} \% \mathrm{Yb}^{3+}$ and $0.4-0.6 \mathrm{~mol} \%$ $\mathrm{Tm}^{3+}$ were prepared by conventional solid-state reaction method using high purity $\mathrm{Yb}_{2} \mathrm{O}_{3}, \mathrm{Tm}_{2} \mathrm{O}_{3}$, and as-synthesized $\gamma$-AlON mixture.
After the preparation, the researchers conducted structure characterization, absorption spectroscopy, and upconversion luminescence spectroscopy of these samples at room temperature. The absorption spectrum of the $\gamma$-AlON: $\mathrm{Yb}$, Tm phosphor exhibits the typical optical transitions of both $\mathrm{Tm}^{3+}$ and $\mathrm{Yb}^{3+}$ which are ${ }^{3} \mathrm{H}_{6} \rightarrow{ }^{3} \mathrm{~F}_{4},{ }^{3} \mathrm{H}_{5},{ }^{3} \mathrm{H}_{4},{ }^{3} \mathrm{~F}_{2,3}$, and ${ }^{1} \mathrm{G}_{4}$ for $\mathrm{Tm}^{3+}$ and ${ }^{2} \mathrm{~F}_{7 / 2} \rightarrow{ }^{2} \mathrm{~F}_{5 / 2}$ for $\mathrm{Yb}^{3+}$. The intense absorption band at $\sim 980 \mathrm{~nm}$ of $\mathrm{Yb}^{3+}$ associated with the transition ${ }^{2} \mathrm{~F}_{7 / 2} \rightarrow{ }^{2} \mathrm{~F}_{5 / 2}$ demonstrates that a $980 \mathrm{~nm}$ laser can efficiently excite the $\mathrm{Yb}^{3+}, \mathrm{Tm}^{3+}$ codoped phosphors. Upconversion luminescence spectra of all samples excited by 